\title{
Ueber Roentgenbefunde am Schlaefebein bei Tumoren.
}

\author{
Von \\ Śaburo Asai. \\ (Ogaki, Japan).
}

Unter den röntgenographisch erkennbaren Veränderungen des Gehörganges spielen die durch Geschwülste hervorgerufenen eine nicht unwichtige Rolle. Allerdings ist die Zahl der bisher in der Literatur diesbezüglich mitgeteilten Beobachtungen eine geringe, wis überhaupt bis vor wenigen. Jahren die Röntgendiagnostik der Affektionen des Gehörganges ein kümmerlich bebautes Feld darstellte. Exst in letzter Zeit mehren sich die Arbeiten auf diesem Gebiete, und zwar sind es vorwiegend die entzündlichen. Frkrankungen ( $\left.L_{s} \mathrm{AW}\right)$, die Verletzungen und dic Missbildungen (Bhwesi) im Bereiche des Gehörganges, deren röntgenographische Darstellung das Interesse in Ansjruch nimmt.

In den folgenden Zeilen soll über 3 Fälle berichtet werden, bei welchen mit Hilfe des Röntgenbildes ausgedehnte, durch T'unioren verursachte Destruktionen im Bereich des Schläfckeins festgestellt werden konnten.

Fall I. betrifft einen $14 \mathrm{j}$. Jüngling, welcher am 24. III. 1914 von. der Universitäts-Ohrenklinik in Wien dem Zentralröntgeninstitut des allgemeinen Krankenhauses (Vorstand Prof. HoLzкnechi) zur Untersuchung überwiesen wurde. Der Patient hatte die Ohrenklinik wegen einer sehr entstellenden Deformität des Kopfes, die sich im Iaufe mehrerer Jahre ganz allmählich ausgebildet hatte, aufgesucht.

Die rechte Schläfegegend zeigte eine starke Vorwölbung, die rechte Ohrmuschel stand wesentlich tiefer als die linke, der rechte Augapfel wies einen beträchtlichen Grad von Exophthalmus, auf. Von Interesse war es, dass die Funktion sowol des rechten Auges wie des rechten Ohres ungestört war. Weit besser als durch Inspektion und Palpation liessen sich die Details der Deformität durch das Röntgenbild 
feststellen. Am übersichtlichsten zeigt die Sagittalaufnahme mit antero-posteriorem Strahlengang (siehe Taf. Fig. ) die am Skelett der rechten Schädelhälfte vorhandenen Anomalien. Was zunächst das SCHÄDELDACH betrifft, so weist dasselbe eine hochgradige Asymmetrie der beiden Temporo-Parietalgegenden auf. Die linke Schädelhälfte zeigt eine annähernd normale Grösse und Form, bezw. eine geringgradige hydrokephale Vergrösserung, wobei ihre Dicke $2-5 \mathrm{~mm}$ beträgt. Die rechte Hälfte des Schädeldaches dagegen zeigt eine faustgrosse, halbkugelige Vorwölbung der Schläfegegend. Die vọgebauchte Partie der Schädelwand ist hochgradig verdünnt, stellenweise fehlt der Knochen vollständig. Die über die vorgewölbte Partie ziehenden äusseren Schädelweichteile zeigen keine Veränderung. Im Bereiche der ScHäDELbasis finden sich gleichfalls rechterseits hochgradige Deformierungen. Der Boden der mittleren Schädelgrube, welcher von dẹr Squama temporalis gebildet wird, ist beträchtlich nach abwärts verdrängt und geht in scharf gekrümmten Bogen in die kurz zuvor beschriebene Vorwölbung der. Seitenwand des Schädeldaches über. Zusammen mit der Pars squamosa ist auch der Jochbogen nach unten und aussen verdrängt. Die genannten Teile der Schädelbasis zeigen ausser der Form- und Lageanomalie keine wesentliche Veränderung ihrer Dicke und Struktur. Was schliesslich die Pars petross des rechten Schläfebeins betrifft, so erscheint dieselbe, abgesehen davon, dass das rechte Felsenbein im Ganzen etwas tiefer steht als das linke, auch schmächtiger als links, wohl infolge Arrosion der Crista petrosa, bezw. der oberen Pyramidenfläche. Im Bereiche der hinteren Schädelgrube zeigt die Schädelbasis keine nennenșerte Formveränderung. Schon auf dieser Aufnahme fällt eine bedeutende Differenz der Umrandungen beider Orbitae auf. Noch deutlicher ist diese Differenz. an der zweiten Sagittalaufnahme mit podero-anteriorem Strahlengang erkennbar. Der rechte Orbitalgang ist nach allen Seiten hin erweitert, so zwar, dass der transversale Durchmesser der rechten Orbita ungefähr $1 \mathrm{~cm}$ grösser ist als der der linken Seite, während der vertikale Durchmessex den der linken Seite etwa um $1.5 \mathrm{~cm}$ übertrifft. Das 
Jochboin der rechten Seite ist verschmächtigt.

Von sonstigen Veränderungen des Gesichts- und des Schädelbasisskeletts zeigt diese Aufnahme folgende Details. Das Orbitaldach und die laterale Orbitalwand erscheinen vollständig destruiert, die mediale Wand (Iamina papiracea), ist gegen die Mittellinie zu verlagert, die untere Orbitalwand herabgedrängt. Infolge der Destruktion der Orbitalwände erscheint das von dem erweiterten Orbitaleingang umrahmte Areal gegenüber der linken Seite aufgehellt. Im übrigen zeigt das Gesichtsskelett keine wesentliche Veränderung: Ober- und Unterkiefer sind im Ganzen schmüchtig, das Nasengerüst normal konfiguriert, die Stirnhöhlen gross, symmetrisch, lufthältig. Die Kieferhöhlen sind von mittlerer Grösse, die rechte steht etwas tiefer als die linke. Beide sind lufthältig.

Eine wünschenswerte Ergänzung des durch die beiden Sagittalaufnahmen gegebenen Befundes bietet die transversale Anfnahme mit sinistro-dextralen Strahlengang (S. Taf. Fig. ). Die zeigt im Bereich des schädeldaches den ausgedehnten Defekt der 'T'emporoParietalregion. Die demselben entsprechende Aufhellung ist nahezu kreisförmig hegrenzt, misst $10 \mathrm{~cm}$ im Durchmesser und ist nur stellenweise noch von knochendichten schattenflecken durchsetzt. Im Bereich der Schädelbasis erkennt man wieder sehr gut die Ausbauchung und Herabdrängung des Bodens der mittleren Schädelgrube. Auch die Sella turcica erscheint erweitert und vertieft. Der Boden der rechten vorderen Schädelgrube (das rechte Orbitaldach) erscheint destruiert. Endlich erkennt man die Tieferstellung und Formveränderung des rechten Felsenboines.

Zusammenfassend lassen sich die im vorliegenden Fall röntgenographisch erkennbaren Formverändurungen folgendermassen darstellen. Entsprechend der Inspektion so aufrällig in die Erscheinung tretenden Ausladung der rechten Scheitel-Schläferegion findet sich eine hochgradige Vorwölbung und Verdünnung des Schädelknochens. Der Boden der rechten mittleren Schädelgrube ist nach ạussen und unten verdrängt, das rechte Felsenbein ist verschmä- 
chtigt und steht tiefer. Die Sella turcica ist beträchtlich erweitert, die Wände der rechten Orbita sind destruiert uud hiedurch der rechtsseitige Exophthalmus verursacht.

Die Formveränderung des Schädels in dem eben beschriebenen Falle stellt ein ganz ungewöhnliches. Vorkommnis dar, insbesondere mit Rücksicht auf die grosse Ausdehnung derselben. Als Ursache derartiger Formveränderungen der Schädelwand kommen hauptsächlich 3 Affektionen in Betracht: 1. der einseitige Hydrokephalus internus, 2. die Meningokele spuria traumatioa, 3. ein grosser intrakranieller Tumor. Was die. Meningokele spuria traumatica (Billroth) betrifft, so versteht man darunter eine nach Frakturen in der frühen Kindheit sich entwickelnde Schädeldeformität, bei welcher ausgedehnte Teile der Schädelwand mangelhaft ostifiziert sind, wobei die verdünnten oder unverknöchert gebliebenen. Teile der Schädelwand meist stärker vorgebaucht sind durch zystische Flüssigkeitsansammlungen, die, an der Aussenfläche oder im Innern des Gehirns liegend, einen abnormen Druck auf die darüber liegende Partie der Schädelwand ausüben (Auvray). Dass ein unilateraler Hydrokephalus intrernus eine lokale Vorbauchung der Tempero-Parietalgegend verursachen kann, hat DANDy bei einem vor kurzem publizierten Fall gezeigt. In ähnlicher Weise wie bei den genannten Afiektionen, jedoch in noch weit ausgedehnterem Masse als bei denselben, kann eine lokale Verdünnung und Vorbauchung der Schädelwand durch intrakranimile Geschwülste hervorgerufen werden, insbesondere dann, wenn dieselben im jugendlichen Alter auftreten, ein langsames Wachstum zeigen und sich flächenhaft zwischen Gehirn und Schädel ausbreiten, wie dies bei Endotheliomen, Fibromen, Angiomen und Sarkomen der Fall sein kann. Auch im vorliegenden Falle dürfte die Schädelabnormität durch eine intrakranielle Geschwulstbildung verursacht sein. Der Fall erinnert einigermassen an eine von Lauber und Schüller*) publizierte Beobachtung, einen 28 j. Mann betreffend, bei welchem sich rechts-

*) Laduber u. Schulder (Zeitschr. f. Augenhlk. 1908, Bd. 19, S. 172) fassen ihren Fall als Lymphengiom der intra- und extrakraniellen Weichteile auf. 
seitiger Exophthalmus, Vorwölbung der rechten Schläfegegend und eine elephantiastische Verdickung der Weichteile der rechten Gesichtshälfte fand, wobei das Röntgenbild im Bereich der rechten Schädelhälfte analoge Knochendestruktionen aufwies, wie in dem hier beschriebenen Fall (Schüller).

Der folgende Fall betrifft eine Patientin mit Karzinom-Metastasen des Schädels.

Fall II. 40 j. Frau, Patientin des Herrn Assistenten K. Gross. Der von demselben verfassten Krankengeschichte entnehmen wir folgende anamnestische Daten: Die Patientin wurde im November 1920 wegen Karzinom der Mammae operiert. Im Sommer 1921 bemerkte sie eine Beule am Hinterkopf, welche ab und zu schmerzhaft war. Anfangs Dezember 1921 trat Doppeltsehen auf, auch starke Kopfschmerzen quälten in letzter Zeit die Patientin.

StA'T. PRÄs. vom 31.12.1921 (Dr. Gross). Kräftige, gut genährte, gesund aussehende Frau. Am Hinterhaupt rechts eine diffuse teigige Sehwellung, die nicht druckempfindlich ist. Dagegen besteht Druckempfindlichkeit der obersten Halswirbel. Puls 80. Nystagmus nach beiden Seiten. Bei Stehen mit geschlossenen Augen Falltendenz nach rechts. Patellarreflex rechts gleich links, beiderseits gesteigert. Bauchdeckenreflex rechts gleich links, Zehenbewegungen rechts etwas weniger ausgiebig.

Augenbefund (Dr. JüNNkr): Beiderseitige, zentral bedingte Abducensparese, besonders rechts. Ohrenbefund (Doz. Ru'r'is): Gehör beiderseits normal. Vestibularbefund normal, bis auf Fehlen des Vorbeizeigens im linken Schultergelenk bei kalorischer Priifung links.

Gelegentlich der am 14.2.1922 erfolgten Untersuchung wirde konstatiert: Rechtes Ohr: Flüsterstimme wird nicht gehört, wol aber lantes Sprechen vor dem Ohr. Die Nervenuntersuchung lisst Steigerung der Reflexe am der rechten Körperhälfte feststellen.

Das Rontgenbits (s. Taf. Fig. ) ergibt: Das Schädeldach ist von normaler Grösse und Form, symmetrisch. Seine Dicke ist betrïchtlich vermehrt. Die der Medianlinie benachbarten Anteile der 
beiden Scheitelbeine sind bis auf $15 \mathrm{~mm}$ verdickt. Die Struktur des Schädeldaches ist fast durchwegs diploetisch. Die Innenfläche des Schädeldaches ist im allgemeinen eben, nur im Bereich des Stimbeins sieht man mehrere halbkugelige Höcker vorspringen, die zumeist haselnussgross sind. An verschiedenen Stellen des Schädeldachs sieht man Aufhellungen des Knochenschattens, mit bogenförmigen, ziemlich unscharfen Umrandungen. Die grösste derartige Aufhellungszone findet sich im Bereich der Schädelhöhe. Sie betrifft die medianen Anteile beider Scheitelbeine und reicht noch ziemlich weit in das linke Scheitelbein hinein. Sie erstreckt sich vorwiegend auf die Diploe, während sie die beiden Grenzlamellen relativ intakt lässt. Eine ähnliche, der Fläche nach weniger ausgedehnte, jedoch die ganze Dikke des Knochens betreffende Destruktion erkennt man an derjenigen Stelle des Hinterhauptes, wo die Palpation eine teigige Schwellung konstatieren kann. Kleinere Aufhellungen von Linsen und Bohnengrösse sieht man im Bereich der restlichen Anteile des Schädeldaches, insbesendere in der Umgebung des Hinterhauptherdes. Im Bereiche der Schädelbasis fällt auf der sagittalen Röntgenaufnahme mit postero-anteriorer Durchleuchtungsrichtung (s. 'Iaf. Fig. ) eine deutliche Differenz der beiden Felsenbeinpyramiden anf. Die linke Pyranide zeigt normale Form und Struktur. Thre Konturen sind innerhalb des Rahmens der Orbita deutlich erkennbar. Die rechte Pyramide erscheint wesentlich kürzer, als die linke, infolge Fehlens ihres medialen, der Felsenbeinspitze entsprechenden Anteils. Der stehengebliebene Anteil der Pyramide grenzt sich mit einem scharfen, zackigen Kontur ab. Infolge der Verkürzung des der rechten Pyramide entsprechenden richattenfeldes ist die mediale Partie der Orbita mit der Fissura orbitalis superior rechts deutlich erkennbar, während diese Partie der linken Orbita links durch den dichten Schatten der Pyramidenspitze iiberlagert erscheint.

Im vorliegenden Fall handelt es sich um eine als Teilerscheinung multiple Metastasierung eines Mamma-Karzinoms in die Schädelwand aufgetretene Destruktion der Felsenbeinspitze. Dieser Ort ist. wie aus 
der Literatur (Manasse) zu ersehen ist, ein Prädilektionsort für Karzinom-Metastasen innerhalb des Schädelbeins:

Der folgende Fall betrifft eine Usur der Pars mastoidea durch ein Cholesteatom.

Fall III. 43 j. Mann, am 28. X. 1919 von der Ohrenklinik mit der Diagnose Cholesteatom der linken Pyramide zur Röntgenuntersuchung überwiesen. Die schräg-seitliche Aufnahme (s. Taf. Fig. ) des linken Schläfebeins ergibt eine vollständig sklerosierte Pars mastoidea, in deren Bereiche ein kreisrunder Aufhellungsherd von 1 I/2 cm Durchmesser erkennbar ist. Der Rand, des dieser Aufhellung entsprechenden Defektes ist im hiutern Anteil des Defektes scharf, während der vordere Rand desselben allmaihlich in den dichten Schatten der Pyramide übergeht.

Mit Rücksicht auf die Grösse, Form und Begrenzung des Defektes sowie auch seine Lokalisation an der Prädilektionsstelle der Cholesteatome (entsprechend der Antrum-Gegend) kann im vorliegenden Falle wol auch aus dem Röntgenbilde die Diagnose "Usur des Schläfebeins durch Cholesteatom" gestellt werden.

Die hier angeführten Fälle reprïsentieren charakteristische Formund Strukturveränderungen des Schläfebeins bei Tumoren, welche teils im Schläfebein selbst entstehen, teils von der Ungebung desselben ilıren Ausgangspunkt genommen haben. In der Röntgen-Literatur finden sich, wie bereits erwähnt, nur wenige hierhergehörige Fälle mitgeteilt. So beschreibt LAw einen Fall von Usur der Pars mastoidea durch ein Cholesteatom, welches grosse Aehnlichkeit aufweist mit dem hier beschriebenen Fall Nr. 3. Ferner beschreibt Law eine Beobachtung von multiplen Defekten im schläfebein infolge von intrakraniellen Zysten. Wiederholt wurden bei Acusticus-Tumoren Usuren im Bereich des Meatus anditorius internus, auf deren röntgenographische Darstellbarkeit zuerst Henschis aufmerksam gemacht hatte, vorgefunden. Geeignete Objekte für die röntgenographische Darstellung sind die Usuren im Bereich der Pyramide bei Aneurysmen der basilaren Arterien, bei grossen T'tumeren der Hypophyse und bei Endotheliomen 
der Dura mater. Bei letzteren können indes auch lokale Hyperostosen des Knochens an der dem Sitze des Tumors entsprechenden Stelle beobachtet werden. Schliesslich kommen die Osteome, Osteosarkome und die durch Ostitis deformans hervorgerufenen hyperostotischen Veranderungen des Schläfebeins für die Röntgendarstellung in Betracht.

\section{Literatur-Verzeichnis.}

ALExANDER, Ohrenkrankheiten im Kindesalter; 1912.

Auvray M., Maladies du crane et de l'enéphale; Paris 1909.

Benesi, Atresie des Gehörganges.

DANDY, Localization or elimination of cerebral tumers by ventriculography; Surgery, Gynoecology and Obstetries. April 1920.

KoERner Otto, Die eitrigen Erkrankungen des Schlafebeins; Wiesbaden 1899.

LAw, Mastoids, Annels of Roentgenology; New Vork 1920.

MaNasse, Handbuch der pathol. Anatomie des menschlichen Ohres; Wiesbaden 1917.

Politzer, Lehrbuch der Ohrenheilkunde; 1908.

SchumLler ARrHUR, Röntgendiagnostik der Erkrankungen des Kopfes; Wien und. Leipzig 1912.

Urbantschitsch V., Lehrbuch der Ohrenheilkunde; Wien 1910. 


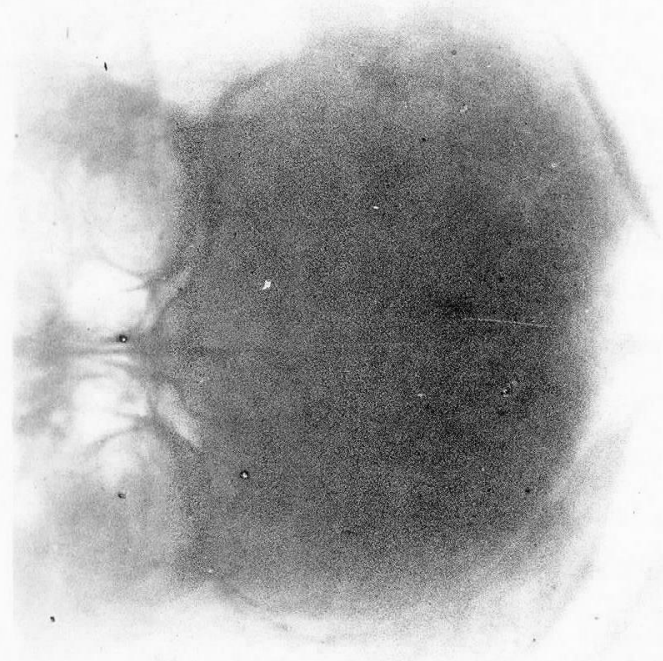

(1)

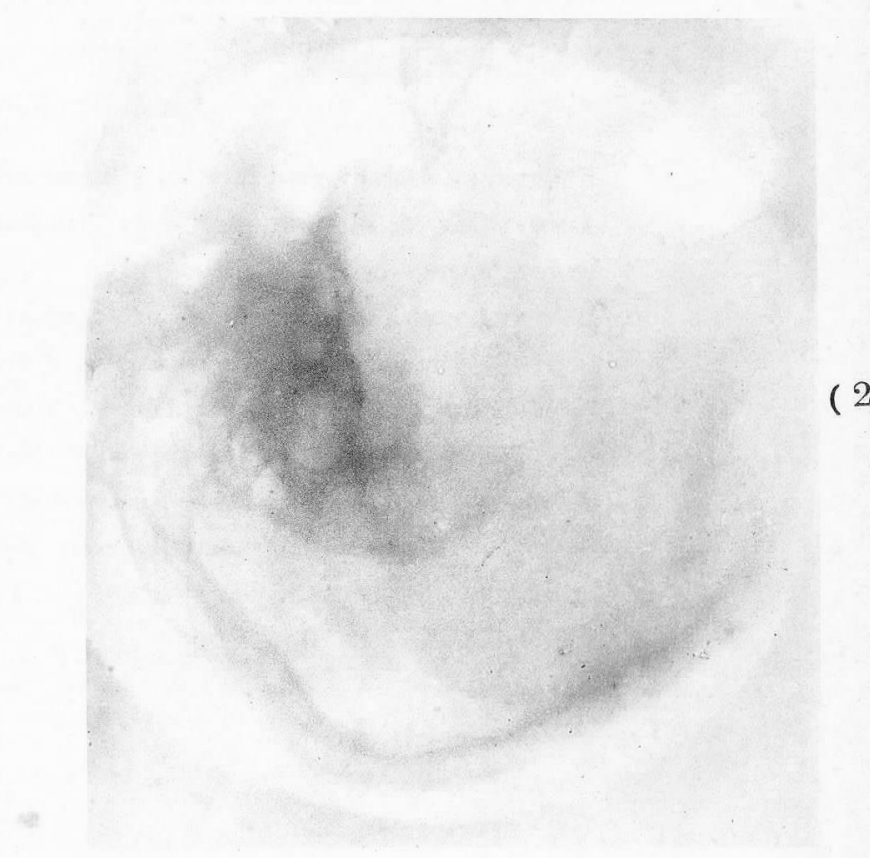

(3)

(1) Carcinom des Schädels.

(Mamma-Ca-Metastase)

(2) Cholesteatom

(3) Endokranieller Tumor 